\title{
Woman and Depression
}

\author{
Shimmy V. $S^{1 *}$
}

\section{ABSTRACT}

Background: woman manifest a wide range of stress responses in their daily life in the personal life at home and job related stress at workplace. It can be in the form of physical disorders ranging from headache, neck ache, shoulder pain, lack of sleep, lack of appetite and in their extreme conditions, it can lead to lack of attention, frustrations, anxiety, depression and to suicidal thoughts. This can interfere their well being and quality of life activities of daily living also. Aim: The research examined the prevalence of depression among young adult woman residing in Kerala State. Research Design: Cross sectional survey design was used. Sample: A sample of 30 people (young adult females) over the age of 18 years participated in the study. Tool used: To assess the depression, CES-D a screening test for depression developed by Radolff (1977)was used. Results: Most of the participants does not have depression, but to a lesser extend mild to moderate depression and possibility of major depression was also found among the participants. Conclusion:. Early screening of depression at regular basis provides intimation towards the woman who need immediate interventions, including counseling facilities and even medical support on right time, protects them from hampering of their functional abilities to a greater extend.

Keywords: Woman, Depression

\section{Woman}

MARCH 2016 - Women's health was highlighted at this year's Commission on the Status of Women (CSW) in New York. WHO was involved in events and side panels addressing a broad range of issues relevant to women's health, including non-communicable diseases (NCDs), nutrition, household energy, and post-rape care.

These events focused on strategies to prevent, manage, and collaboratively mobilize on NCDs and mental health. And also invited attention of health researchers and practitioners to discuss how to best work together to communicate and address important health issues, particularly NCDs. The dynamic discussions that occurred within these events were key in identifying barriers and opportunities to collaborative work in addressing health and social inequalities experienced across women.

\footnotetext{
${ }^{1} \mathrm{PhD}$ Research Scholar, School of Behavioral Sciences, MG University, Kerala, India

*Responding Author

(C) 2016 I V Shimmy; licensee IJIP. This is an Open Access Research distributed under the terms of the Creative Commons Attribution License (http://creativecommons.org/licenses/by/2.0), which permits unrestricted use, distribution, and reproduction in any Medium, provided the original work is properly cited.
} 


\section{Depression}

Depression is a common mental disorder, characterized by sadness, loss of interest or pleasure, feelings of guilt or low self-worth, disturbed sleep or appetite, feelings of tiredness, and poor concentration.

Depression can be long-lasting or recurrent, substantially impairing an individual's ability to function at work or school or cope with daily life. At its most severe, depression can lead to suicide. When mild, people can be treated without medicines but when depression is moderate or severe they may need medication and professional talking treatments.

Depression is a disorder that can be reliably diagnosed and treated by non-specialists as part of primary health care. Specialist care is needed for a small proportion of individuals with complicated depression or those who do not respond to first-line treatments.

The literacy rate for women in Kerala stands at 87.86 per cent, compared to the Indian average of 54 per cent. In fact, women's life expectancy, maternal mortality rate, infant mortality rate and fertility rate all compare favorably with those of many wealthy developed countries. The suicide rate among women is reported to be twice the national average. The suicide rate of women in Kerala at 27 per $100,000^{1}$, compared to the national average (for both men and women) of 10.With education women become more aware of their potential. But tradition, culture, family, society and state - all patriarchal in structure and ideology - have short-changed women.

Depression is predicted to be the world's leading cause of disability by the year $2030^{2}$. It has long been observed that women are about twice as likely to become clinically depressed ${ }^{3}$. The report on Global Burden of Disease estimates the point prevalence of unipolar depressive episodes to be $1.9 \%$ for men and $3.2 \%$ for women, and the one-year prevalence has been estimated to be $5.8 \%$ for men and $9.5 \%$ for women. It is estimated that by the year 2020 if current trends for demographic and epidemiological transition continue, the burden of depression will increase to $5.7 \%$ of the total burden of disease and it would be the second leading cause of disability-adjusted life years (DALYs), second only to ischemic heart disease ${ }^{4}$.Women and depression is holding a relationship of much interest over the last two decades. As more and more women enter the work force, they are increasingly exposed not only of the same work environment as men, but also to unique pressure created by multiple roles and conflicting expectations. Modernity brought women education in its wake and she changed the arena of activity. She stepped out of the house and joined service like man. Now she got admiration, equality and opportunity. But she was supposed to take to this job as an additional responsibility. She not expected to shrink household work. This brought problems like strain and depression.

There is a lack of proper surveys, epidemiological or community based, on the prevalence of depression among young adult women, most of whom will be married in the Kerala cultural 
scenario. The purpose of this study was to find out the prevalence of depression among adult woman in Kerala.

\section{METHODOLOGY:}

The study aims to screen the depression among the young adult woman in Kerala. Cross sectional design was used in the study. Convenient Sample of 30 young adult woman residing in Kerala has been taken. Data was collected from the participants through home visits. Inclusion criteria: young adult woman residing in Kerala, without any other physical or psychiatric illnesses. Exclusion criteria: less than 18years of age and having a confirmed diagnosis of any physical or psychiatric illness and taking treatment for that.

\section{Tools Used}

Demograhic data: for collecting the informations, age, education, occupation, marital status, family income per year, no of children and presence of elderly bed ridden member at home.

The Center for Epidemiological Studies-Depression (CES-D), originally published by Radloff in 1977, is a 20-item measure that asks caregivers to rate how often over the past week they experienced symptoms associated with depression, such as restless sleep, poor appetite, and feeling lonely. Response options range from 0 to 3 for each item $(0=$ Rarely or None of the Time, 1 = Some or Little of the Time, 2 = Moderately or Much of the time, $3=$ Most or Almost All the Time). Scores range from 0 to 60 , with high scores indicating greater depressive symptoms.

The CES-D also provides cutoff scores (e.g., 16 or greater) that aid in identifying individuals at risk for clinical depression, with good sensitivity and specificity and high internal consistency (Lewinsohn, Seeley, Roberts, \& Allen, 1997) ${ }^{5}$.

\section{Procedure:}

Participants were informed about the study and the associated confidentiality. Participants were clearly explained that they could discontinue their participation if they felt to do so at any point of time during the assessment. Informed consent was taken from the participants, and the tools were administered individually. Data was collected and analyzed by using appropriate descriptive statistical measures.

\section{RESULT:}

1. The demographic data ( Table 1) shows that participants were in their young adulthood, aged between 20-29 years, $N=30$. Age( Table 1)

\begin{tabular}{|l|l|l|}
\hline Age & No of participants & Percentage (\%) \\
\hline $18-21$ & 0 & \\
\hline $22-25$ & 11 & 36.67 \\
\hline $26-29$ & 19 & 63.33 \\
\hline
\end{tabular}


2. Education (Table 2) Among the participants most of them were graduates $N=17$ (56.67\%), but postgraduates were $N=8(26.67)$ and undergraduates were $N=5(16.67)$. Education (Table 2)

\begin{tabular}{|l|l|l|}
\hline Education & No of participants & Percentage(\%) \\
\hline Under graduation & 5 & 16.67 \\
\hline Graduation & 17 & 56.67 \\
\hline Post graduation & 8 & 26.67 \\
\hline
\end{tabular}

3. Occupation, Among participants it is observed that maximum number of participants are engaged in private jobs, $N=14(46.67)$, second majority was housewives $N=12(40 \%)$,but 4 participants $N=4(13.33 \%)$ had Govt job, Occupation(Table 3)

\begin{tabular}{|l|l|l|}
\hline Occupation & No of participants & Percentage(\%) \\
\hline House wives & 12 & 40 \\
\hline Private Job & 14 & 46.67 \\
\hline Govt job & 4 & 13.33 \\
\hline
\end{tabular}

4. Marrital status, Most of the participants are married $N=24(80 \%)$, and the rest are $N=6(20 \%)$ unmarried., Marrital status(Table 4)

\begin{tabular}{|l|l|l|}
\hline Marrital status & No of participants & Percentage(\%) \\
\hline Married & 24 & 80 \\
\hline Unmarried & 6 & 20 \\
\hline
\end{tabular}

5. Family income per year, Among the members maximum number of participants belongs to upper class $N=19(63.33 \%)$, upper middle class is $N=8(26.67 \%)$ and middle class $N=3(10 \%)$., Family income per year(Table 5)

\begin{tabular}{|l|l|l|}
\hline Income/year & No of participants & Percentage(\%) \\
\hline Less than 10,032 p.a (BPL) & Nil & \\
\hline $\begin{array}{l}\text { 10,033 to 20,040 p.a. (low } \\
\text { middle class) }\end{array}$ & Nil & 10 \\
\hline $\begin{array}{l}\text { 33,241 to 33,240 p.a. } \\
\text { (middle class) }\end{array}$ & 3 & 26.67 \\
\hline $\begin{array}{l}33,241 \text { to 66,840 p.a. } \\
\text { (upper middle class) }\end{array}$ & 8 & 63.33 \\
\hline Above 66,840 (upper class) & 19 & \\
\hline
\end{tabular}

6. No of children (among married $N=24)$, Among the participants( $N=24$ married woman) maximum number $N=16(66.67 \%) h a s ~ 2$ child, 5 of the participant has single child $N=5(20.83 \%)$ and the rest $N=3(12.50 \%)$ has three or more child., No of children(Table 6)

\begin{tabular}{|l|l|l|}
\hline No of Children & No of participants having children & Percentage(\%) \\
\hline 1 & 5 & 20.83 \\
\hline 2 & 16 & 66.67 \\
\hline$\geq 3$ & 3 & 12.50 \\
\hline
\end{tabular}


7. Presence of elderly bed ridden member at home, Maximum number of participants does not have any bed ridden family member to be looked after, $N=27(90 \%)$ and the 3 participants has bedridden member at home to be looked after, $N=3(10 \%)$, Presence of elderly bed ridden member at home(Table 7)

\begin{tabular}{|l|l|l|}
\hline $\begin{array}{l}\text { No of elderly bed ridden } \\
\text { member at home }\end{array}$ & No of participants & Percentage(\%) \\
\hline yes & 3 & 10 \\
\hline No & 27 & 90 \\
\hline
\end{tabular}

\section{Depression}

Maximum number of participants screened, does not have depression, which is clinically evident $\mathrm{N}=27(90 \%)$, Mild to moderate depression for 1 member $\mathrm{N}=1,(3.33 \%)$ and 2 participant has the possibility of major depression ,N=2(6.67\%).

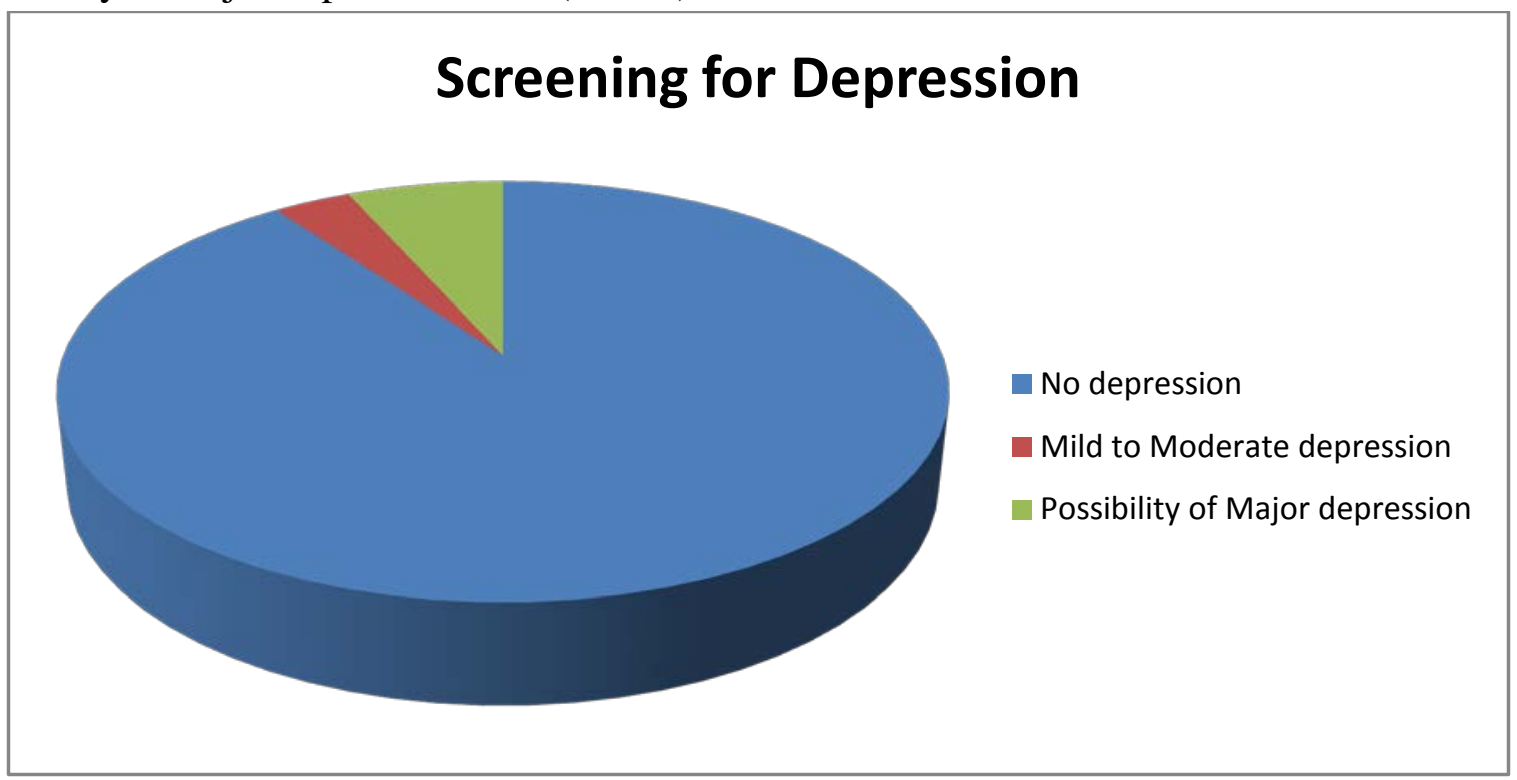

\section{DISCUSSION:}

Studies have reported that economic and interpersonal relationship difficulties, partner violence, sexual coercion by the partner as the common causal factors related to development of depression in general and depression during antenatal and postnatal period ${ }^{6,7,8,9,10}$.It was also reported that poverty (low income and having difficulty in making ends meet), being married as compared with being single, use of tobacco, experiencing abnormal vaginal discharge and reporting a chronic physical illness were associated with risk of developing a common mental disorder $^{11}$. Women as a group have also received considerable attention with regard to risk factors for development of depressive disorders. In an incidence study of common mental disorders $^{11}$.

\section{CONCLUSION:}

Nearly one tenth of the women screened positive for depression , which is at a high magnitude keeping in view the prevalence rate of the country and the global estimates. Early screening for 
depression among adolescents in school level and family counseling availability for marital disharmony, domestic violence and addiction makes a role in the decreased prevalence of depression .Early detection and treatment options for mental illnesses at Primary health centre levels throughout the state along with the reproductive health care facilities and workplace wellbeing clinics are essential for the reduction in the prevalence of depression among woman in Kerala.

\section{REFERENCES}

1. National Crime Records Bureau. Accidental deaths and suicides in India-2007. National Crime Records Bureau. Ministry of Home Affairs, Government of India. http://ncrb.nic.in/ADSI2007/home.html

2. WFMH. Depression: A global Crisis. World Mental Health Day, October 10 2012. World Federation for mental Health 2012

3. Kessler RC. Epidemiology of women and depression. Journal of Affective Disorders. 2003 Mar;74(1):5-13.

4. Lopez AD, Mathers CD, Ezzati M, Jamison DT, Murray CJ. Global Burden of Disease and Risk Factors. Washington: The World Bank; 2006.

5. Lewinsohn, P.M., Seeley, J.R., Roberts, R.E., \& Allen, N.B. (1997). Center for Epidemiological Studies-Depression Scale (CES-D) as a screening instrument for depression among community-residing older adults. Psychology and Aging, 12, 277- 287.

6. Chandran M, Tharyan P, Muliyil J, Abraham S. Post-partum depression in a cohort of women from a rural area of Tamil Nadu, India Incidence and risk factors. British Journal of Psychiatry. 2002;181:499-504.

7. Pereira B, Andrew G, Pednekar S. The explanatory models of depression in low income countries: Listening to women in India. Journal of Affective Disorders. 2007;102:20918.

8. Patel V, Rodrigues M, DeSouza N. Gender, Poverty, and Postnatal Depression: A study of mothers in Goa, India. American Journal of Psychiatry. 2002;159:43-7.

9. Rodrigues M, Patel V, Jaswal S, De Souza N. Listening to mothers: Qualitative studies on motherhood and depression from Goa, India. Social Science and Medicine. 2003;57:1797-806.

10. Verma D, Chandra PS, Thomas T, Carey MP. Intimate partner violence and sexual coercion among pregnant women in India: Relationship with depression and post-traumatic stress disorder. Journal of Affective Disorders. 2007;102:227-35.

11. Patel V, Kirkwood BR, Pednekar S, Weiss H, Mabey D. Risk factors for common mental disorders in women Population-based longitudinal study. British Journal of Psychiatry. 2006;189:547-55

How to cite this article: V Shimmy (2016), Woman and Depression, International Journal of Indian Psychology, Volume 3, Issue 3, No. 8, DIP: 18.01.139/20160303, ISBN: 978-1-36512176-0 